\title{
Inosine Alters Gene Expression and Axonal Projections in Neurons Contralateral to a Cortical Infarct and Improves Skilled Use of the Impaired Limb
}

\author{
Laila Zai, ${ }^{1,2}$ Christina Ferrari, ${ }^{1}$ Sathish Subbaiah, ${ }^{1,4}$ Leif A. Havton, ${ }^{5}$ Giovanni Coppola, ${ }^{5}$ Stephen Strittmatter, ${ }^{6}$ \\ Nina Irwin, ${ }^{1,2,4}$ Daniel Geschwind, ${ }^{5}$ and Larry I. Benowitz ${ }^{1,2,3,4}$ \\ ${ }^{1}$ Laboratories for Neuroscience Research in Neurosurgery and ${ }^{2}$ F. M. Kirby Neurobiology Center, Children's Hospital Boston, and ${ }^{3}$ Program in \\ Neuroscience and ${ }^{4}$ Department of Surgery, Harvard Medical School, Boston, Massachusetts 02115, ${ }^{5}$ Program in Neurogenetics, Department of Neurology, \\ University of California, Los Angeles, Los Angeles, California 90095, and 'Department of Neurology, Yale University School of Medicine, New Haven, \\ Connecticut 06510
}

\begin{abstract}
Recovery after stroke and other types of brain injury is restricted in part by the limited ability of undamaged neurons to form compensatory connections. Inosine, a naturally occurring purine nucleoside, stimulates neurons to extend axons in culture and, in vivo, enhances the ability of undamaged neurons to form axon collaterals after brain damage. The molecular changes induced by inosine are unknown, as is the ability of inosine to restore complex functions associated with a specific cortical area. Using a unilateral injury model limited to the sensorimotor cortex, we show that inosine triples the number of corticospinal tract axons that project from the unaffected hemisphere and form synaptic bouton-like structures in the denervated half of the spinal cord. These changes correlate with improved recovery in animals' ability to grasp and consume food pellets with the affected forepaw. Studies using laser-capture microdissection and microarray analysis show that inosine profoundly affects gene expression in corticospinal neurons contralateral to the injury. Inosine attenuates transcriptional changes caused by the stroke, while upregulating the expression of genes associated with axon growth and the complement cascade. Thus, inosine alters gene expression in neurons contralateral to a stroke, enhances the ability of these neurons to form connections on the denervated side of the spinal cord, and improves performance with the impaired limb.
\end{abstract}

\section{Introduction}

In the US alone, over 350,000 people who survive a stroke each year suffer persistent sensorimotor and/or cognitive deficits that significantly impair daily living. Current treatments are limited to the use of thrombolytic agents and physical therapy. Strategies to improve outcome by limiting secondary injury, although successful in animal studies, have shown little benefit clinically (Dobkin, 2003). Another strategy for improving outcome after stroke might be to stimulate the reinnervation of brain regions that have lost their normal inputs. After focal brain injury, undamaged neurons undergo changes in their dendritic arbors (Allred and Jones, 2004; Hsu and Jones, 2006; Papadopoulos et al., 2006) and axonal projections (Carmichael et al., 2001; Car-

\footnotetext{
Received Jan. 26, 2009; revised March 29, 2009; accepted May 18, 2009.

This work was supported by the National Institutes of Health (NIH; Grant R01 NS047446), the Miriam and Sheldon G. Adelson Medical Research Foundation, and Alseres Pharmaceuticals. We thank the Developmental Disabilities Research Center of Children's Hospital (supported by NIH Grant P30 HD018655) for use of the histology and image analysis cores, Charles Vanderberg for instruction and providing access to the Laser Capture Core Facility of the Harvard Center for Neural Discovery, David Goldberg for help in establishing the experimental model, Jeremy DavisTurak and Fuying Gao for assistance with microarray analyses, Carlie Dice for help with immunostaining, Judith Li, Haleh Hashemi, and Melissa Hootstein for behavioral training and testing, and Hui-Ya Gilbert for help with photography.

Correspondence should be addressed to Dr. Larry Benowitz, Laboratories for Neuroscience Research in Neurosurgery, Children's Hospital Boston, 300 Longwood Avenue, Boston, MA 02115. E-mail: larry.benowitz@ childrens.harvard.edu.

D0I:10.1523/JNEUROSCI.0414-09.2009

Copyright $\odot 2009$ Society for Neuroscience $\quad$ 0270-6474/09/298187-11\$15.00/0
}

michael, 2003; Dancause et al., 2005; Nudo, 2006) that enable them to assume some of the functions formerly mediated by the damaged areas (Nudo, 2007). Thus, treatments that enhance anatomical reorganization may help improve functional outcome after stroke.

Factors that limit anatomical reorganization after brain injury include the low intrinsic potential of most CNS neurons to extend axons, and the many inhibitory proteins associated with myelin and the perineuronal net. In a unilateral stroke model, interfering with the inhibitory protein Nogo or one of its receptors, $\mathrm{NgR}$, enables corticospinal neurons (CSNs) on the intact side of the brain to sprout axon collaterals into the denervated side of the spinal cord and improves animals' ability to use the affected paw (Papadopoulos et al., 2002; Emerick et al., 2003; Lee et al., 2004; Cafferty and Strittmatter, 2006).

A complementary way to promote rewiring after stroke is to augment neurons' intrinsic growth state (Kawamata et al., 1997). The purine nucleoside inosine activates Mst3b, a protein kinase that is part of a cell-signaling pathway that regulates axon outgrowth (Irwin et al., 2006). Inosine stimulates axon outgrowth from several types of neurons in culture (Zurn et al., 1988; Benowitz et al., 1998; Irwin et al., 2006), and in vivo, it enhances the ability of neurons contralateral to an injured hemisphere to extend axon collaterals into denervated parts of the brainstem and spinal cord (Chen et al., 2002; Smith et al., 2007). In the present study, we use a more refined injury model to show that inosine 
Table 1. Experimental groups

\begin{tabular}{|c|c|c|c|c|}
\hline Expt. & Animals per group & Rationale & Survival time & Analyses \\
\hline I & 12 & Functional recovery, anatomical reorganization & 4 weeks & Behavioral testing, anatomical tracing \\
\hline ॥ & 6 & Effect of stroke alone & 4 weeks & Anatomical tracing \\
\hline III & 6 & Effect of inosine in the absence of brain injury & 4 weeks & Anatomical tracing \\
\hline IV & 12 & Persistence of recovery & 8 weeks & Behavioral testing \\
\hline V & 6 & Short-term reorganization & 2 weeks & Anatomical tracing \\
\hline VI & $4-6$ & Changes in gene expression & 1 week & Laser-capture microdissection, microarrays \\
\hline VII & $4-8$ & Changes in protein levels & 1 week & Immunohistochemistry, quantitative analysis \\
\hline
\end{tabular}

Expt., Experiment.

affects gene expression in CSNs contralateral to a stroke, enhances these neurons' ability to extend new axons and form synaptic-like structures on the denervated side of the spinal cord, and almost completely restores fine motor control with the forepaw previously controlled by the damaged hemisphere.

\section{Materials and Methods}

All procedures were performed in accordance with National Institutes of Health Guidelines with the approval of Children's Hospital Animal Care and Use Committee. In all, 118 adult Sprague Dawley rats (275-300 g; Charles River) were used in the experiments outlined in Table 1 . The first part of the study investigated the effect of inosine on brain reorganization and behavioral outcome after unilateral injury centered in the forepaw motor area. We then investigated whether unilateral stroke per se causes neurons in the undamaged hemisphere to sprout corticospinal tract (CST) axon collaterals; whether inosine induces axonal sprouting in the absence of brain injury; whether the functional effects of inosine persist after treatment ends; and whether inosine alters CST reorganization at an earlier time point. The final part of the study examined the effects of inosine at the molecular level.

Stroke surgery. With the exception of animals in part III and one group of controls in parts VI and VII, all rats sustained unilateral infarcts in the sensorimotor cortex using the photothrombotic model of focal ischemia (Markgraf et al., 1993). Briefly, rats were anesthetized with a combination of ketamine $(75 \mathrm{mg} / \mathrm{kg}$ ) and Domitor (medetomidine; $0.5 \mathrm{mg} / \mathrm{kg}$ ), and a $15 \mathrm{~mm}$ skin incision was made at the midline rostral to the posterior suture. A craniotomy was performed over the sensorimotor cortex using a hand-held drill to open a window that spanned mediolaterally between the sagittal sinus and temporal ridge, and rostrocaudally between bregma $+2.5 \mathrm{~mm}$ and bregma $-3.5 \mathrm{~mm}$. The photosensitive dye Rose Bengal was injected into the femoral vein, a fiber-optic cable connected to a xenon light source was centered over the craniotomy, and the exposed region was illuminated for $30 \mathrm{~min}$. A green filter fitted over the bulb restricted illumination to $\sim 525 \mathrm{~nm}$, a wavelength that excites Rose Bengal and causes it to release free radical species. The subsequent damage to endothelial cells in exposed portions of the cortical vasculature causes platelet aggregation, resulting in severe focal ischemia. As shown below, infarcts typically had a diameter of $6-7 \mathrm{~mm}$ and were restricted to cortical tissue and some underlying white matter. Uninjured control animals in parts III, VI, and VII were generated using the same surgical procedure but without photo-activation of the injected Rose Bengal.

Animals with strokes were randomly assigned to receive a continuous infusion of either saline (0.9\%, Baxter Scientific) or inosine $(50 \mathrm{~mm}$ in saline, Sigma-Aldrich) into the lateral ventricle of the uninjured hemisphere using osmotic minipumps $(0.25 \mu \mathrm{l} / \mathrm{h}$, Alzet model 2004, Durect Corporation). Catheters were placed $1.4 \mathrm{~mm}$ lateral and $0.8 \mathrm{~mm}$ caudal to bregma on the uninjured hemisphere within 30 min of surgery. Because CSF drug concentrations presumably require several hours to achieve steady-state levels when delivered via slow-releasing osmotic pumps, all animals received a $25 \mu \mathrm{l}$ intraventricular bolus of the appropriate agent before pump placement at the time of injury. Pumps were tucked between the shoulder blades and infusion needles were secured onto the cranium with a silicon-based glue. The incision was closed with silk sutures and cleaned with betadine and ethanol pads. For $72 \mathrm{~h}$ after surgery, animals received twice-daily, subcutaneous injections of $\mathrm{Bu}$ prenex (buprenorphine; Reckitt Benkiser Pharmaceuticals) for pain management. Animals in the second experiment had strokes but did not receive intraventricular infusions.

Behavioral testing. Functional recovery was evaluated using a skilled forelimb-reaching task on which animals were trained before surgery. In the first part of the study, animals ( $N=12$ per group) were tested at 7, 14 , 21 , and $28 \mathrm{~d}$ after surgery before being injected intracranially with the anatomical tracer. In the fourth part of the study $(N=12$ per group), minipumps were removed after 4 weeks and testing was continued weekly for another 4 weeks. All testing was done by an experimenter blind to the animals' treatments.

The behavioral task requires rats to reach with either paw through a narrow slit in a Plexiglas box, grasp a banana-flavored food pellet (BioServ) from a platform, and bring it successfully to the mouth (Allred and Jones, 2004; Luke et al., 2004). Three days before and during preoperative training, animals were maintained on a restricted diet of banana-flavored pellets to remove novelty-induced hesitation and to increase motivation. Rats were trained 30-60 min per day with each paw for 2 weeks or until they reached a baseline performance of 20-30 successful reaches in a 2 min period. At the end of the training period but before surgery, animals were tested for the number of pellets successfully grasped and consumed in two 2 min trials, making sure that they were motivated and stress free. The average of these two tests became the "baseline" score, to which subsequent scores were normalized. This enabled us to account for possible individual differences in motivation and competence as a biasing factor for overall performance. Performance was recorded only if rats retrieved a minimum of 25 pellets within the 2 min interval. In postsurgical testing, we likewise scored performance only when animals were fully engaged and performing the task at a relatively consistent level. Within each session, animals spent 10-20 min in the apparatus, during which they were tested twice with each paw (alternating) for $2 \mathrm{~min} /$ session. Scores from the unimpaired paw were used to assess animals' engagement, motivation, and overall behavioral competence. Data were analyzed using a regular two-way ANOVA. Bonferroni's post-test was used to compare data sets.

Anterograde tracing of crossing fibers. Animals in part I of the study were anesthetized after the final testing period, the infusion needle and pump were removed, and a craniotomy was performed over the uninjured sensorimotor cortex (SMC). The anterograde tracer biotinylated dextran amine (BDA: Invitrogen: 10,000 molecular weight, 10\% w/v in sterile saline) was injected stereotaxically at depths of $0.5,1.0$, and $2.0 \mathrm{~mm}$ below the cortical surface at 18 standardized points distributed over the sensorimotor cortex, as determined by the Paxinos and Watson (1998) rat brain atlas (supplemental Fig. 1, available at www.jneurosci.org as supplemental material) (70 nl per injection; Nanoject, Drummond Scientific). Two weeks later, animals were reanesthetized and perfused transcardially with $0.9 \%$ saline followed by $4 \%$ paraformaldehyde. The brain and spinal cord were dissected and postfixed overnight in $4 \%$ paraformaldehyde, followed by $10 \%$ and $30 \%$ sucrose solutions over the next few days. Tissue was embedded in OCT Tissue Tek Medium (Sakura Finetek) and frozen on dry ice. Forty-micrometer free-floating sections were cut in the coronal plane on a Frigo-Jung 8500 cryostat. Free-floating spinal cord sections were used to visualize the trajectory of CST axons using avidin-biotin complex conjugated to horseradish peroxidase (Vectastain ABC Kit; Vector Laboratories), followed by Vector SG (Vector Laboratories) as a chromagen. Sections were mounted on precoated slides and lightly counterstained with eosin to distinguish gray and white 
matter boundaries. Six to ten sections spanning a distance of $1.2 \mathrm{~mm}$ were examined in each case and quantified for (1) BDA-labeled axon profiles $\geq 40 \mu \mathrm{m}$ in length within the dorsal funiculus on the denervated side of the spinal cord (i.e., ipsilateral to the BDA injection); (2) BDAlabeled axons $\geq 40 \mu \mathrm{m}$ in length in the gray matter of the denervated side of the spinal cord, and (3) BDA-labeled axons $\geq 200 \mu \mathrm{m}$ in length on the denervated side. Axon length was measured in the transverse plane. The number of axons was normalized by the density of CST staining within the intact dorsal funiculus to adjust for possible differences in labeling between animals. The latter values were obtained by densitometric measures of the intact CST and converting density values to a scale of $0-1$ $(0=$ density in the denervated side of the dorsal funiculus, $1=$ density in the case with the most intense labeling). Three coronal sections through the rostral cervical cord were used to obtain the normalization values for each animal. Average numbers of axons, normalized for labeling density, were calculated and converted to axons per millimeter of spinal cord. Bouton-like structures were identified under a $100 \times$ oil objective as areas $\geq 2 \times$ the thickness of the axon shaft. Counts were performed in a 0.25 $\mathrm{mm}^{2}$ box aligned with laminae VI/VII, and were not extrapolated to any volumetric totals.

Animals in part II were used to investigate the effect of stroke on CST reorganization in the absence of treatment. We created left-sided strokes in six animals, while carrying out sham surgery (Rose Bengal injections, craniotomy, no illumination) in another six animals. As above, BDA was injected into multiple sites in the right forelimb motor area 4 weeks later and animals were prepared for histology after another 2 weeks. Animals in group III were use to investigate whether inosine would induce sprouting in the absence of brain injury. These animals ( $N=6$ per group) underwent sham surgery, received either inosine or saline for 4 weeks as above, and were then prepared for anatomical tracing of CST fibers that originate on one side of the brain and project to the ipsilateral cervical enlargement. Animals in group IV ( $N=12$ per group) were used to investigate whether the functional benefits of inosine persist after treatment ends. Animals were prepared as in the first set of experiments, but at the completion of inosine delivery, behavioral testing continued weekly for another 4 weeks. Animals in group $\mathrm{V}(N=6$ per group) were used to investigate whether inosine-induced changes in CST projections could be seen as early as 2 weeks after injury.

Determination of lesion severity. Sections through the telencephalon of animals in group I were cut at $10 \mu \mathrm{m}$, mounted onto slides, and stained with Crystal Violet to determine the extent of the lesions. Sections were scanned using a high resolution Epson Perfection 3490 PHOTO scanner. The cross-sectional area of the injured and uninjured hemisphere of each section was determined using NIH ImageJ software. Lesion area was determined by subtracting the area of tissue remaining in the injured hemisphere from that in the uninjured hemisphere in sections spaced $250 \mu \mathrm{m}$ apart spanning the full rostrocaudal extent of the lesion. Lesion volume was extrapolated from these data. Lesions were redrawn onto standard sections from a rat brain atlas (Paxinos and Watson, 1998). Representations of the injury were created from tracings of scanned sections in Adobe Photoshop.

Effects of inosine on gene expression. The final parts of this study investigated molecular events induced by inosine in the population of neurons that give rise to the undamaged corticospinal tract. To identify these neurons, animals were anesthetized with a combination of ketamine and Domitor (medetomidine) and a laminectomy was performed at the cervical level of the spinal cord (C2-C4). Alexa Fluor 488-conjugated cholera toxin B subunit (CTB; $300 \mathrm{U} / \mu \mathrm{l}$ in sterile saline) was stereotaxically injected into four sites lateral to the corticospinal tract. Injections $(1.3 \mu \mathrm{l})$ were spaced $1 \mathrm{~mm}$ apart on the rostrocaudal axis and were $0.5 \mathrm{~mm}$ to the left and right of the midline, just flanking the CST. CTB is avidly taken up by fibers of passage (Chen and Aston-Jones 1995), and thus these injections are expected to label layer 5 neurons that project to the level of the injection site and below, including the forepaw region. After allowing 2 weeks for transport of the tracer, we performed either sham surgery with no treatment or stroke surgery combined with saline or inosine delivered via minipumps, as described above. Seven days later, animals were decapitated under transient gas anesthesia, brains were removed and rinsed in cold RPMI medium, and a tissue block containing the sensorimotor cortex was dissected (based on stereotaxic coordinates) and placed in OCT Tissue Tek Medium (Sakura Finetek) on dry ice within 5 min of death. Ten-micrometer sections were cut onto precleaned Gold Seal RITE-ON glass slides (Gold Seal Products), placed on dry ice, and rapidly stored at $-80^{\circ} \mathrm{C}$ until ready to use.

Laser-capture microdissection and microarray analysis. Slide-mounted sections were thawed and dehydrated in RNase-free ethanol gradients and xylene. Retrogradely labeled, fluorescent cortical pyramidal cells in the forelimb area of the undamaged hemisphere were individually captured using the Arcturus VERITAS system. Cells ( $\geq 500)$ were collected from each animal and stored in Arcturus extraction buffer at $-80^{\circ} \mathrm{C}$. Total RNA was extracted from cells using the Micro-to-Midi TotalRNA Purification System (Invitrogen) and double-amplified using the TargetAmp 2-Round Aminoallyl-aRNA Amplification kit 1.0 (Epicenter). Amplified RNA was checked for average fragment length using the Agilent RNA 6000 Nano LabChip kit (Agilent Technologies) and then biotinylated and hybridized $(1 \mu \mathrm{g})$ on Illumina RatRef-12 Expression BeadChip arrays (Illumina), querying the expression of $>22,000$ RefSeqcurated rat transcripts. Results were obtained from a total of 15 samples representing 6 untreated controls, 4 animals with stroke treated with saline, and 5 animals with stroke treated with inosine. Slides were processed and scanned with Illumina BeadStation platform according to the manufacturer's protocol.

Raw data were analyzed using Bioconductor packages [www. bioconductor.org (Gentleman et al., 2004)]. Low-level quality-control analysis was performed using interarray Pearson correlation and clustering based on variance. To further control for RNA integrity, three indirect measures of comparable RNA integrity were run across samples.

Agilent Bioanlyzer Nanochips were run on the double-amplified RNA samples to insure uniform RNA degradation, as determined by average fragment size. A subset of samples were run on both Illumina and Affymetrix arrays to insure that $5^{\prime}$ to $3^{\prime}$ ratios were comparable.

Finally, the detection scores-a measure of detection ranging between 0 (not detected) and 1 (strongly detected) provided by the Illumina BeadStudio software-were compared across samples and showed no difference across samples (supplemental Table 2, available at www.jneurosci. org as supplemental material). Two arrays (one saline- and one inosinetreated) were outliers and were excluded from the analysis. Data were normalized using quantile normalization, and analysis of differential expression was performed using a linear model fitting [LIMMA package (Smyth, 2005)]. Differentially expressed genes were classified according to gene ontology using Bioconductor packages and online tools (DAVID, http://david.abcc.ncifcrf.gov/). Pathway analysis was performed using Ingenuity Pathway Analysis (Ingenuity Systems).

Immunohistochemistry and quantitation of activated caspase 3 and other proteins. Animals in the last part of the study were used to visualize the effect of inosine on the expression of several proteins. This set included four normal controls, eight rats with stroke and saline infusions, and eight with stroke and inosine infusions, prepared as described above. One week after surgery, animals were killed, perfused with saline and paraformaldehyde, and their brains were postfixed and prepared for histology. Sixteen-micrometer cortical sections were mounted onto slides and allowed to dry at room temperature for $2 \mathrm{~h}$. To visualize caspase activation, sections were washed in PBS, boiled for $20 \mathrm{~min}$ in citrate buffer, blocked in $5 \%$ BSA, and incubated overnight in a $2 \%$ BSA solution containing an antibody to activated caspase 3 (1:500, Abcam). The following day, slides were rinsed in PBS and incubated with Alexa Fluor 568-conjugated goat anti-rabbit secondary antibody (1:500, Invitrogen) for $2 \mathrm{~h}$ at room temperature. Slides were rinsed in PBS and coverslipped with Fluorosave mounting medium (Invitrogen). Immunopositive profiles bordering the lateral aspect of the lesion were quantified in a $1 \mathrm{~mm}^{2}$ field at bregma 1.7 $\mathrm{mm}$ in five animals selected randomly from each group. As stereological methods were not used, these profile counts are strictly focal in the information they convey. To insure that between-group differences were not caused by changes in the diameter of these profiles, we measured the cross-sectional areas of stained profiles in 20 consecutive objects in 4 sections/case.

Immunohistochemistry and quantitation of complement proteins and metallothionein. Slide-mounted sections were postfixed in 10\% buffered 
formalin, blocked in 5\% BSA, and incubated overnight in goat anit-C1q (1:500, Sigma), goat anti-C3 (1:500, Cappel), or rabbit antimetallothionein (1:50, Santa Cruz Biotechnology) primary antibodies overnight. After a rinse, sections were incubated in appropriate Alexa Fluor 488-conjugated secondary antibodies, rinsed, and coverslipped using Fluorosave mounting medium (Invitrogen). Complement intensities were quantified by digital thresholding of images using NIH ImageJ. Metallothionein-positive cells were quantified by individual counts in a $1 \mathrm{~mm}^{2}$ field bordering the lateral aspect of the lesion at bregma 1.7 $\mathrm{mm}$. As with caspase counts, these numbers are from profile counts and represent cell densities at this level only, not volumetric or threedimensional measures.

\section{Results}

\section{Lesion placement and size}

We used a photothrombotic stroke model to generate unilateral ischemic lesions centered in the forelimb motor area of the cortex. The infarct area was determined using stereotaxic coordinates and was verified by histological analysis. Lesions were restricted to one hemisphere and included the caudal forelimb area of the primary motor cortex along with varying amounts of adjacent cortex (Fig. 1; supplemental Figs. 1, 2, available at www.jneurosci.org as supplemental material). Lesion size varied among animals in each group, but there were no systematic between-group differences [lesion size (in pixels) $=85.4 \pm 8.2 \mathrm{~d}$ (mean \pm SEM) for the saline-treated group and $81.1 \pm 8.6$ for the inosine-treated group: difference not significant]. This finding suggests that inosine was not neuroprotective. This conclusion is further supported by the absence of any difference between inosine- and saline-treated cases in the number and size of profiles expressing activated caspase-3, as quantified focally in a standardized area on the periphery of the stroke (caspase-3 profiles in saline-treated group $=$ $11.8 \pm 1.1$, and in inosine treated group $=11.9 \pm 1.5$ ). Cannulas delivering inosine or saline, placed into the lateral ventricle caudal to the forelimb motor area, did not cause excessive tissue damage (supplemental Fig. 3, available at www.jneurosci.org as supplemental material).

\section{Inosine promotes the sprouting of CST axons from the intact hemisphere into the denervated side of the spinal cord}

To investigate the effect of inosine on CST reorganization, we injected BDA into multiple sites in the forelimb motor area of the undamaged hemisphere at the completion of behavioral testing (supplemental Figs. 1, 2, available at www.jneurosci.org as supplemental material). After allowing 2 weeks for BDA to be transported down the length of the CST, animals were killed and prepared for histology. Relative to controls, animals treated with inosine showed a threefold increase in the number of CST fibers that entered the denervated dorsal funiculus from the undamaged hemisphere $(p<0.01)$ (Fig. $\left.2 a, a^{\prime}, e\right)$. Within the gray matter on the denervated side of the cord, inosine induced a 2.5 -fold increase in the number of labeled fibers $\geq 40 \mu \mathrm{m}$ in length (Fig. $2 f)(p<0.01)$ and increased the number of lengthy axons $(>200$ $\mu \mathrm{m}) 3.5$-fold (Fig. $2 g)(p<0.01)$. Supplemental Figure 4 (avail-

\section{Saline}

\section{Inosine}

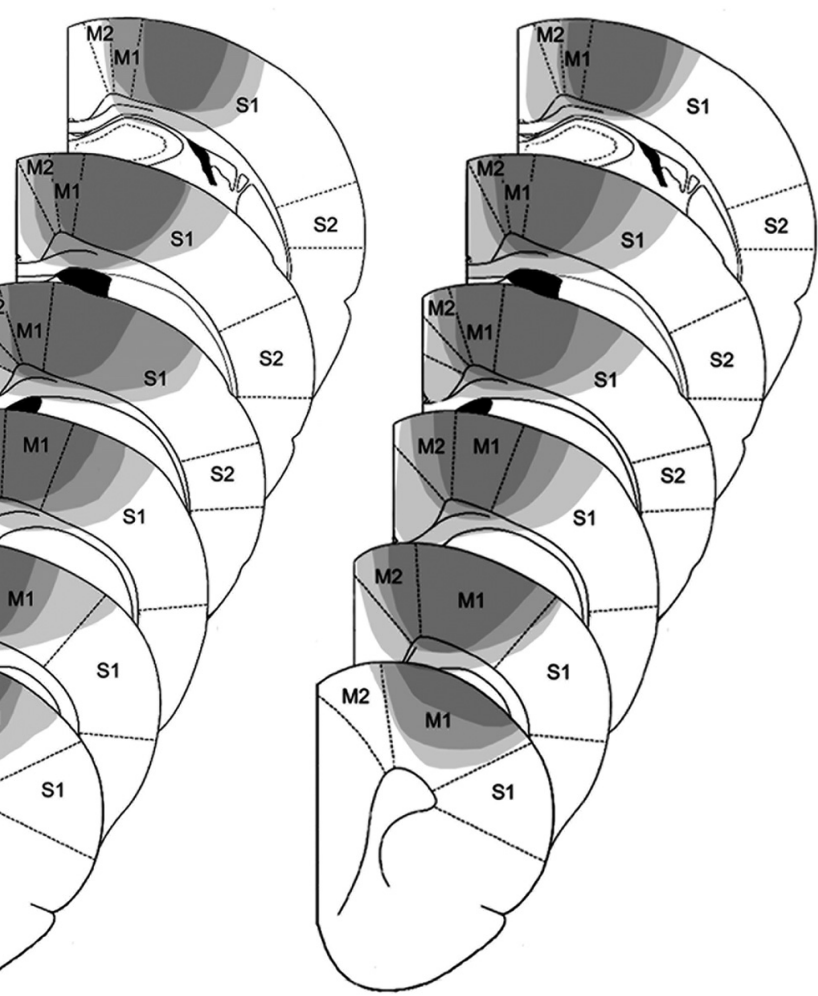

Figure 1. Unilateral brain damage after focal ischemia. Extent of the smallest (dark gray), average (medium gray), and largest (light gray) lesions in saline- (left) and inosine- (right) treated animals 4 weeks after induction of focal ischemia. M1, M2, Primary and secondary motor areas; $S 1$, S2, primary and secondary somatosensory areas.

able at www.jneurosci.org as supplemental material) shows an example of axon crossing at the midline.

At high magnification, labeled CST axons showed numerous bouton-like structures, local swellings that were $\geq 2 \times$ the width of the axons (Fig. $2 b, b^{\prime}$ ). In the spinal cord, similar bouton-like swellings of intracellularly labeled terminal axonal arbors have been shown to correspond to synapses at the electron microscopic level (Lagerbäck et al., 1981; Havton and Kellerth, 1987). Both en passant and terminal boutons were visualized (Fig. 2). Quantifying the density of these structures within a $0.25 \mathrm{~mm}^{2}$ box spanning laminae VI and VII using a $100 \times$ oil objective revealed that inosine tripled the number of synaptic bouton-like structures (Fig. 2h).

Inosine did not alter CST reorganization in the absence of brain injury. To investigate this question, animals underwent sham surgeries (craniotomies, Rose Bengal injections, no photoactivation) and received inosine or saline into the lateral ventricle of the right hemisphere for 4 weeks. We then labeled one hemisphere with BDA and prepared animals for histology 2 weeks later. Inosine- and saline-treated animals showed similarly small numbers of labeled CST axons in the spinal gray matter ipsilateral to the labeled hemisphere $(60 \pm 6.7$ axons/mm for saline-treated cases, and $77.5 \pm 9.0$ for inosine-treated cases: $t=1.56, \mathrm{df}=11$, $P \sim 0.15$ ), indicating that inosine does not stimulate CST axons to sprout in the absence of brain injury and target denervation. To investigate whether stroke per se causes significant anatomical reorganization, animals in experiment III (Table 1) underwent unilateral brain injury but received no further treatment. Four weeks later, the undamaged hemisphere was labeled with BDA and, after allowing another 2 weeks for BDA transport, animals 

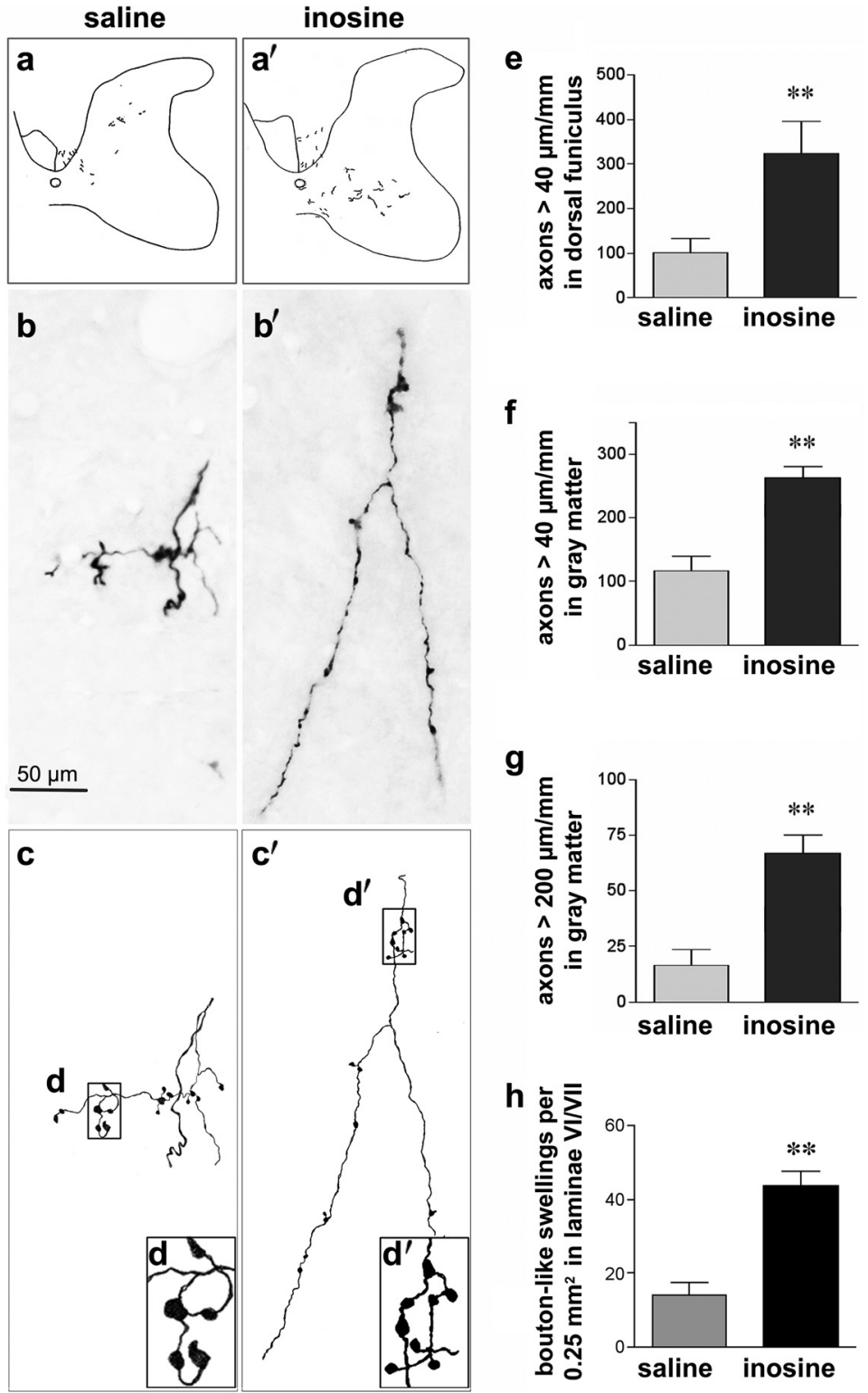

Figure 2. Inosine enhances CST sprouting on the denervated side of the spinal cord. $\boldsymbol{a}, \boldsymbol{a}^{\prime}$, Low-magnification camera lucida drawings of BDA-labeled CST fibers that originate in the uninjured hemisphere and project to the side of the spinal cord denervated by unilateral cortical injury. Rats were treated with either saline $(\boldsymbol{a})$ or inosine $\left(\boldsymbol{a}^{\prime}\right) \cdot \boldsymbol{b}, \boldsymbol{b}^{\prime}$, High-magnification photomicrographs of fibers in the gray matter of saline- $(\boldsymbol{b})$ and inosine- $\left(\boldsymbol{b}^{\prime}\right)$ treated rats. $\boldsymbol{c}, \boldsymbol{c}^{\prime}$, Camera lucida tracings of the fibers in $\boldsymbol{b}$ and $\boldsymbol{b}^{\prime} . \boldsymbol{d}, \boldsymbol{d}^{\prime}$ (insets), Higher magnification views of bouton-like structures. $\boldsymbol{e}, \boldsymbol{f}$, Quantitation of ipsilaterally projecting (ST fibers $\geq 40$ $\mu \mathrm{m}$ in length in the transverse plane in the denervated dorsal funiculus and gray matter, respectively. $\boldsymbol{g}$, Quantitation of ipsilaterally projecting CST fibers $\geq 200 \mu \mathrm{m}$ in length in the denervated gray matter. $\boldsymbol{h}$, Quantitation of bouton-like swellings on fibers projecting to the ipsilateral spinal gray matter visualized under a $100 \times$ oil objective. Results in $\boldsymbol{e}-\boldsymbol{g}$ are normalized by the intensity of staining in the intact CST and are reported as number of labeled axons per mm length of spinal cord. ${ }^{* *}$ Difference between groups significant at $p<0.01$. Error bars represent SEM.

were prepared for histology. Animals with strokes showed $\sim 5$ times more CST fibers that projected from the unaffected hemisphere into the ipsilateral cervical gray matter than shamoperated controls $(40 \pm 16$ axons $/ \mathrm{mm}$ without stroke vs $217.5 \pm$ 57.2 with stroke, $p<0.001)$. Together, these data suggest that inosine augments a naturally occurring reorganization of CST fibers after stroke, but does not alter CST organization in the absence of brain injury.

To examine the time course of CST reorganization, a separate group of animals was generated ( $N=6$ per group) in which CST axons arising from the intact hemisphere were labeled 2 weeks after stroke, rather than 4 weeks. No significant differences were found in the number of BDA-labeled CST fibers between inosine- and saline-treated cases (data not shown).

\section{Inosine improves skilled use of the denervated forepaw}

By using a photothrombotic injury model, we were able to limit injury to the primary motor area and surrounding neocortex with only modest subcortical damage. This enabled us to investigate recovery of skilled behavior with a minimum of nonspecific functional deficits. All animals were trained to criterion with both paws before surgery, and the presurgical scores were used to normalize their scores after stroke. In the first week after stroke, all animals suffered a dramatic drop in their ability to retrieve food pellets with the paw contralateral to the infarct (Fig. 3a). Saline-treated animals initially showed almost no ability to grasp the food pellets, although performance recovered to $35-40 \%$ of their preoperative level over the next 3-4 weeks. The performance of inosine-treated animals was superior to that of controls at 1 week $(p<0.01)$ and became increasingly so over time. By week four, inosine-treated animals were performing at $\sim 80 \%$ of their baseline levels (Fig. 3a: difference from controls significant at $p<0.01$ ). The experimental and control groups both performed normally with the unaffected paw (Fig. 3b), indicating that the deficits seen with the impaired paw are not attributable to changes in motivation or overall activity levels.

\section{Persistent effects of treatment on functional recovery}

A separate set of animals was used to investigate whether the functional effects of inosine would persist after treatment ended. Animals were treated and tested for 4 weeks as before, then tested for an additional 4 weeks after minipumps were removed. As before, saline-treated animals showed almost no ability to retrieve food pellets in the first week and recovered to $\sim 40 \%$ of their preoperative level by weeks three to four. No further improvements were seen at later time points (Fig. 4a). In contrast, inosine-treated animals continued to perform well and even improve over time, going from $\sim 70 \%$ of baseline at week four to $\sim 80 \%$ at week eight. The performance of inosine-treated animals was significantly better than that of saline-treated controls from week two on $(p<0.05$ for weeks two to six, $p<0.01$ for weeks seven and eight). No differences were detected in animals' performance with the unimpaired paw (Fig. $4 b$ ). The results of this study confirm the effects of inosine seen in the first part of the study, and show that these effects persist for at least a month after treatment ends. 
a

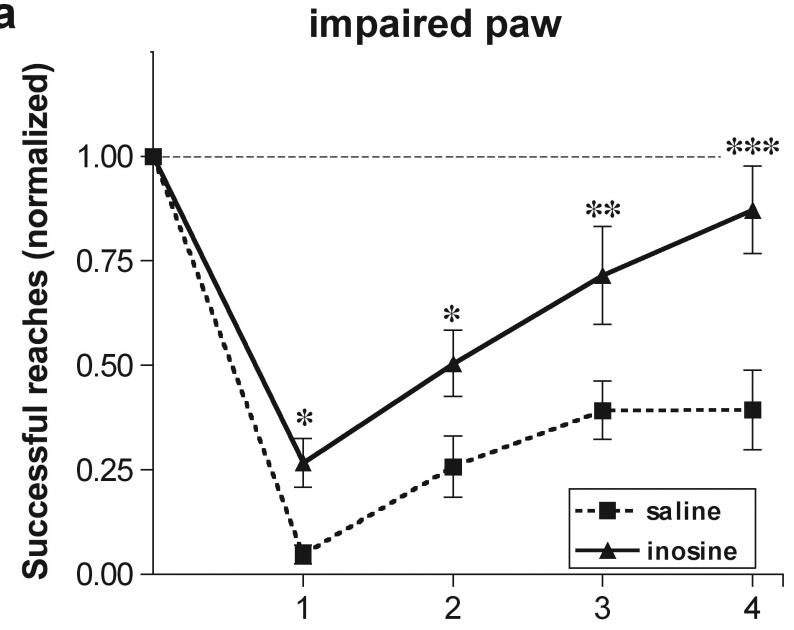

b

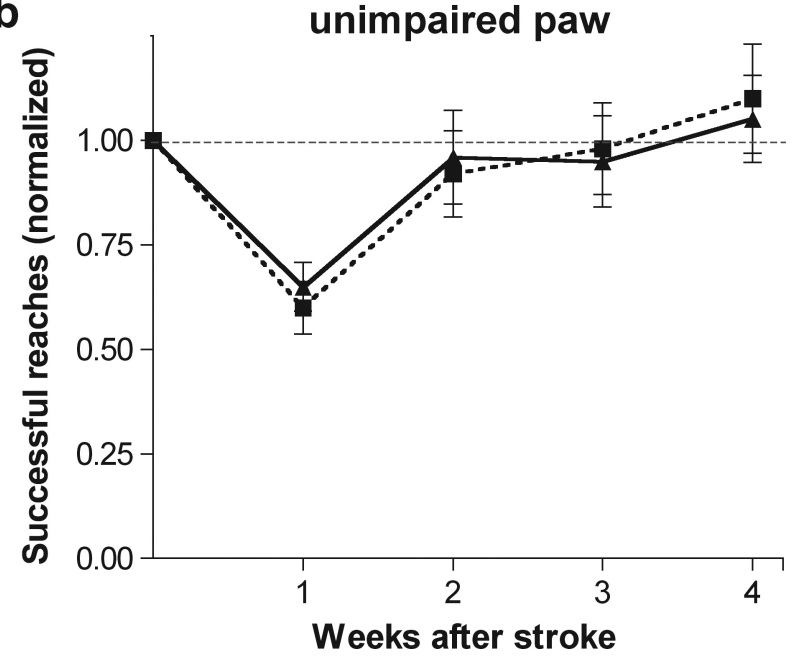

Figure 3. Inosine enhances functional recovery after stroke. Animals were trained to retrieve food pellets through a restricted opening with either paw before surgery and were then tested weekly beginning 1 week later by a blinded observer. Scores are reported as percentage of preoperative performance. $\boldsymbol{a}$, Inosine improves functional recovery using the impaired paw. $\boldsymbol{b}$, Animals show normal performance with the unimpaired paw after 2 weeks regardless of treatment. ${ }^{*},{ }^{* *}$, ${ }^{* * *}$ Differences significant at $p<0.05, p<0.01, p<0.001$, respectively. Error bars represent SEM.

\section{Inosine alters gene expression in neurons contralateral to the lesion}

To investigate the effects of inosine at a molecular level, we used laser-capture microdissection (LCM) to isolate CSNs on the side of the brain contralateral to the stroke $7 \mathrm{~d}$ after surgery and treatment. mRNA from each animal was analyzed separately using Illumina microarrays. Resulting signal intensities ranged from $<100$ to $>30,000$ and showed good reproducibility, with $R$ values (Pearson's correlation) varying from 0.93 to 0.98 between biological replicates. Of the $\sim 22,000$ genes represented on the arrays, stroke and/or inosine treatment caused significant changes in the expression of 636 genes using the following criteria: (1) average signal intensity $\geq 400$ in at least one condition and (2) change significant at $p \leq 0.01$ (supplemental Table 1, available at www.jneurosci.org as supplemental material). Eighty-four percent of these changes resulted from stroke per se (293 decreases in expression and 242 increases) (Fig. 5a). Analysis of the stroke-induced changes using Ingenuity Pathways Analysis soft-
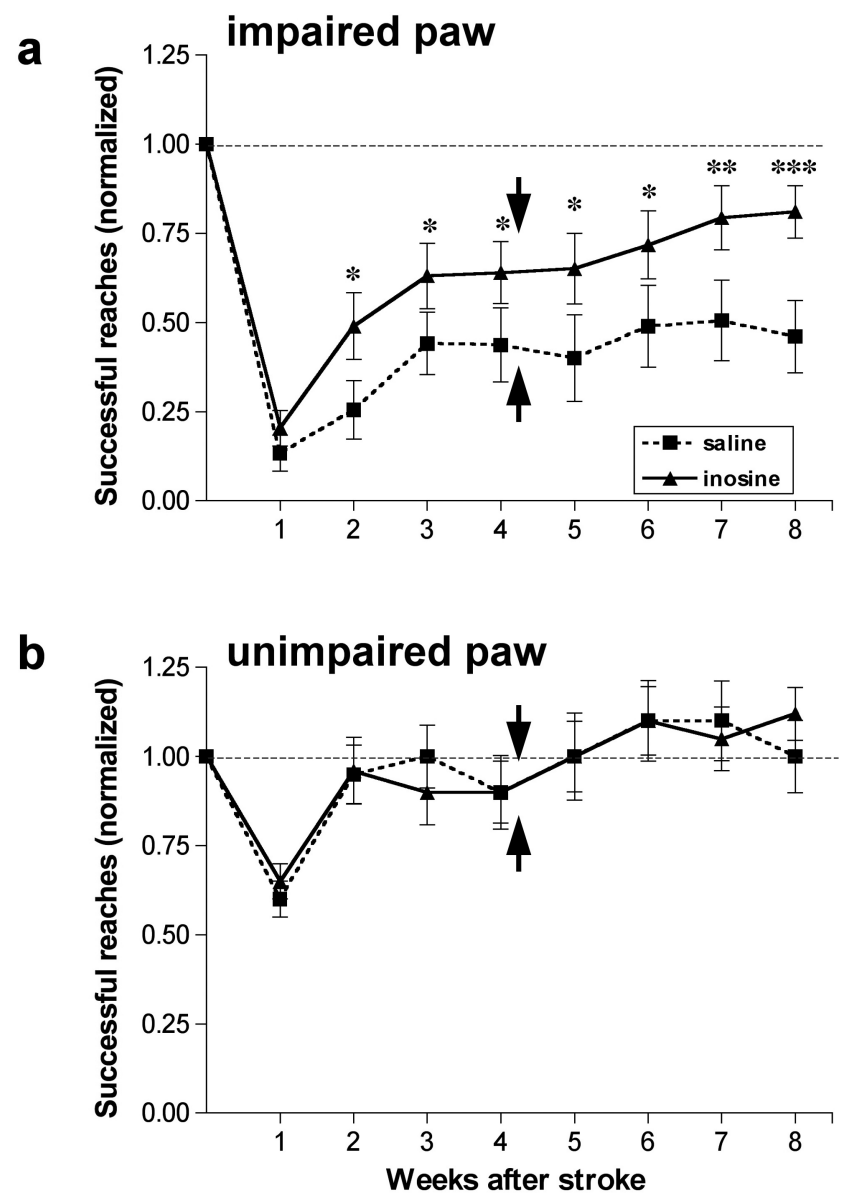

Figure 4. Functional improvements persist after the cessation of treatment. $\boldsymbol{a}, \boldsymbol{b}$, Animals were treated as in Figure 3 but were tested for an additional 4 weeks after treatments ended with either the paw contralateral to the stroke $(\boldsymbol{a})$ or the unaffected paw $(\boldsymbol{b})$. Performance with the affected paw failed to improve after $3-4$ weeks in saline-treated animals, but remained high and even tended to improve after inosine treatment ended. $\boldsymbol{b}$, Performance with the paw ipsilateral to the stroke is unaffected by treatment. ${ }^{*},{ }^{* *},{ }^{* * *}$ Differences between groups significant at $p<0.05, p<0.01, p<0.001$, respectively. Arrows indicate time of pump removal.

ware revealed an over-representation of genes involved in protein ubiquitination $\left(p<10^{-3}\right)$, mostly a downregulation of particular ubiquitination factors (ube4A, ube2M, ube2E3), proteosomal proteins ( $p s m C 5$, psmB5, psmB4, psmA7, hspA8), and heat shock protein ( $h s p$ )-70; genes encoding proteosomal proteins psmB6 and -8 showed increased expression. Also over-represented were genes associated with mitochondrial dysfunction. Stroke caused a significant upregulation of several components of the complement cascade $(c 1 q \beta, c 1 q \gamma, c 2, c 3)$.

Inosine showed two distinct effects: it attenuated most of the stroke-induced changes and induced the expression of a distinct set of genes (Fig. $5 a-d$ ). The effect of inosine in attenuating stroke-induced changes can be seen in the "heat map" of Figure $5 a$, where the intensity of many of the changes induced by stroke (stroke/saline vs control) is diminished by inosine treatment. This effect can also be visualized in the scatter plot of Figure $5 b$. For each gene that is significantly affected by stroke $(p<0.01)$, we plotted the magnitude of the change resulting from stroke along the $x$-axis $\left(\log _{2}\right.$ ratio of expression in animals with stroke treated with saline vs normal controls) and the magnitude of the change resulting from inosine on the $y$-axis $\left(\log _{2}\right.$ ratio of expression in inosine- vs saline-treated cases after stroke). The majority of points cluster around a trend line with a slope of -0.49 and an 
a

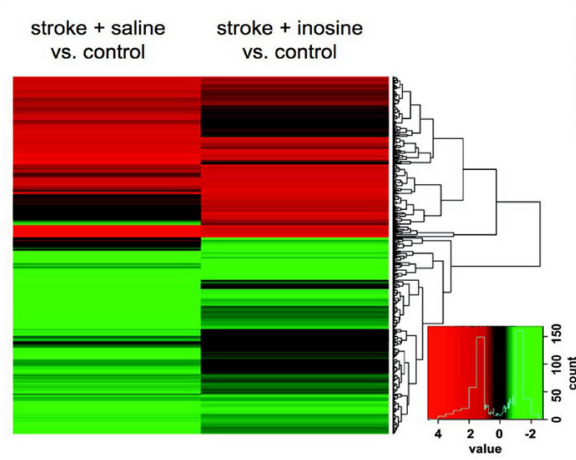

b

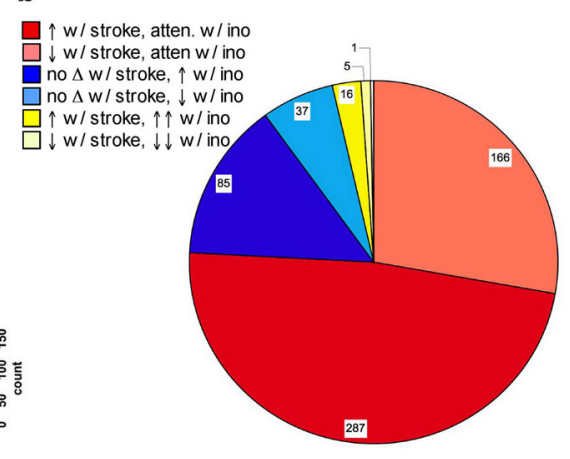

C
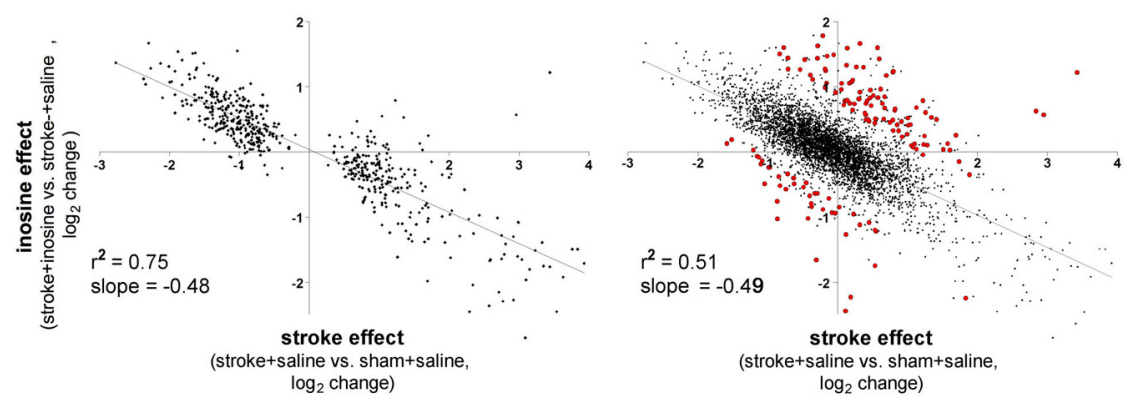

Figure 5. Inosine alters gene expression in corticospinal neurons contralateral to the stroke. $\boldsymbol{a}$, Heat-map showing patterns of changes induced by stroke alone (stroke + saline) and by inosine treatment after stroke (stroke + inosine). Only genes with statistically significant $(p<0.01$ ) changes $\geq 1.7 \times$ above or below baseline level of expression are shown. Inset shows color scheme for the magnitude of changes. $\boldsymbol{b}$, Pie chart showing numbers of genes exhibiting different patterns of change after stroke. Most changes follow the trend of being upregulated or downregulated after stroke and attenuated (atten.) by inosine (ino). A smaller number of genes are not significantly changed by stroke but are either upregulated or downregulated by inosine, and an even smaller number are changed by stroke and changed further in the same direction by inosine. c, Scatter diagram showing effect of inosine in attenuating stroke-induced changes. Data include only those genes whose expression is significantly altered by stroke. Data are plotted as the $\log _{2}$ change in expression after stroke in animals treated with inosine versus saline ( $y$-axis) against the $\log _{2}$ change in expression after stroke alone (and treatment with saline) versus normal controls. Trend line has a slope of -0.49 and correlation coefficient of 0.87 , signifying that inosine attenuates most of the stroke-induced changes. $\boldsymbol{d}$. Scatter diagram similar to that of $\boldsymbol{c}$, but showing all data points. Points that fall significantly off the trend line ( $p<0.01$, red dots) correspond to genes that are affected by inosine over and above attenuating the stroke-induced changes.

$R$ value of 0.87 ( $p<0.0001$ ), indicating that inosine strongly attenuates the changes caused by stroke: a slope of -1 would signify that inosine fully restores changes resulting from stroke to baseline, whereas a more shallow slope or more scatter would indicate a lesser effect of inosine in attenuating the effects of stroke.

The second effect of inosine is represented by the genes that lie at a significant distance from the trend line through the data (Fig. $5 c$ ). The magnitude of this second effect was calculated as the difference between the observed value for the inosine:saline ratio after stroke and the expected value along the trend line of Figure $5 c$. Of the genes that were significantly upregulated by inosine over and above attenuating the effect of stroke, $30 \%$ were also found to be upregulated in retinal ganglion cells stimulated to regenerate their axons in the mature rat optic nerve (Fischer et al., 2004). This amount of overlap is significant at $p<10^{-7}$. Growthrelated genes selectively upregulated by inosine include those encoding tissue inhibitor of metalloproteinase $(\operatorname{timp} 1)$, metallothinonine, and galectin 3 (Table 2), although not others that are associated with optic nerve regeneration, e.g., gap43 and sprrla. The other striking effect of inosine was to increase expression of proteins in the complement cascade, including $c 1 q \alpha, c l q \beta, c 1 q \gamma$, $\mathrm{c} 1 \mathrm{~s}, \mathrm{c} 2, \mathrm{c} 3, \mathrm{c} 4 \mathrm{~B}$, adipsin, serping 1 , and $c f b$ (complement factor B) (Table 3$)$. This set of changes is highly significant $\left(p<10^{-11}\right)$.
To verify that the between-group differences seen in gene expression were not caused by differences in mRNA degradation, we examined RNA stability using several methods. Mean detection scores, a measure of the genes that were detected in the various samples, did not differ among unoperated controls (supplemental Table 2, available at www.jneurosci.org as supplemental material), animals with strokes treated with inosine, and animals with strokes treated with saline. These groups also did not differ when we compared average fragment lengths in doubly amplified RNA samples, as analyzed by Agilent Bioanlyzer Nanochips. We also ran a subset of our samples on both Illumina and Affymetrix arrays, and computed the $5^{\prime}$ to $3^{\prime}$ ratio, finding comparable ratios in all sets.

To investigate whether some of the changes seen in the microarray study translate into differences at the protein level, we performed immunohistochemistry for $\mathrm{C} 1 \mathrm{q}, \mathrm{C} 3$, and metallothionein (study VII). Quantitation of complement staining intensities and counts of metallothionein-positive profiles showed that the inosine-induced changes seen in the microarrays data are also evident at the protein level (Fig. 6). For C1q, inosine increased staining intensity by $75 \%$ in layer 5 of the undamaged hemisphere (average grain pixel density $=3231 \pm 519$ for saline-treated cases and $5654 \pm 894$ for inosine-treated cases: difference significant at $p<0.05)$. For C3, inosine increased staining intensity by $37 \%$ (average grain pixel density $=1002 \pm 101$ for salinetreated cases and $1377 \pm 101$ for inosine: difference significant at $p<0.05)$. Finally, for metallothionine, inosine increased the number of positively staining profiles per field by $28 \%$ (14.0 \pm 1.5 for saline-treated animals and $17.9 \pm 0.8$ for inosine-treated cases).

\section{Discussion}

After a unilateral stroke centered in the forelimb motor region, continuous delivery of inosine altered gene expression in corticospinal neurons (CSNs) of the undamaged hemisphere, stimulated some of these neurons to form axon collaterals and synaptic-like structures on the denervated side of the spinal cord, and restored animals' ability to use the impaired forelimb in a task that would normally have required the lesioned area.

\section{Mechanisms of action}

Inosine induces several types of neurons to extend axons in culture, including those of the embryonic cortex (Zurn and Do, 1988; Benowitz et al., 1998; Irwin et al., 2006). Inosine diffuses across the cell membrane and activates Mst3b, a Ste20-like protein kinase that plays a central role in the signal transduction pathway through which trophic factors induce axon outgrowth. Knock-down of Mst3b expression or expression of a dominant- 
Table 2. Genes that are upregulated by inosine in CSNs contralateral to cortical injury and in retinal ganglion cells undergoing axon regeneration in vivo

\begin{tabular}{|c|c|c|c|c|}
\hline \multirow[b]{2}{*}{ Probe } & \multirow[b]{2}{*}{ Symbol } & \multirow[b]{2}{*}{ Definition } & \multicolumn{2}{|c|}{ Fold induction } \\
\hline & & & $\operatorname{CSMNs}^{a}$ & $\mathrm{RGCs}^{b}$ \\
\hline ILMN_53325 & LOC498335 & (P) Small inducible cytokine B13 precursor (CXCL13) & 11.42 & 3.97 \\
\hline ILMN_70335 & $\mathrm{A} 2 \mathrm{~m}$ & $\alpha$-2-Macroglobulin & 4.10 & 2.36 \\
\hline ILMN_62559 & Timp1 & Tissue inhibitor of metalloproteinase 1 & 3.74 & 13.00 \\
\hline ILMN_51277 & LOC305633 & (P) Antxr2 protein & 3.13 & 4.82 \\
\hline ILMN_60046 & Serping1 & Serine (or cysteine) peptidase inhibitor, clade G, member 1 & 2.93 & 4.63 \\
\hline ILMN_48088 & Ifitm3 & (P) Interferon induced transmembrane protein 3 & 2.74 & 1.99 \\
\hline ILMN_55502 & C1qg & Complement component 1, q subcomponent, $\gamma$ polypeptide & 2.73 & 2.62 \\
\hline ILMN_53575 & Mt1a & Metallothionein & 2.69 & 6.02 \\
\hline ILMN_58058 & Lcp1 & (P) Lymphocyte cytosolic protein 1 & 2.68 & 2.07 \\
\hline ILMN_60003 & Cd68 & (P) CD68 antigen & 2.62 & 3.34 \\
\hline ILMN_60037 & Lgals3bp & Lectin, galactoside-binding, soluble, 3 binding protein & 2.60 & 3.03 \\
\hline ILMN_68224 & Gfap & (P) Glial fibrillary acidic protein & 2.46 & 5.10 \\
\hline ILMN_57422 & Serpinb1a & (P) Serine (or cysteine) proteinase inhibitor, clade B, member 1a & 2.41 & 2.93 \\
\hline ILMN_55731 & Bzrp & Benzodiazepine receptor, peripheral & 2.38 & 2.43 \\
\hline ILMN_47707 & Aif1 & Allograft inflammatory factor 1 & 2.33 & 2.33 \\
\hline ILMN_69642 & Arpc1b & Actin related protein $2 / 3$ complex, subunit $1 B$ & 2.31 & 2.48 \\
\hline ILMN_59412 & Emp3 & Epithelial membrane protein 3 & 2.20 & 2.16 \\
\hline ILMN_48069 & Crabp2 & Cellular retinoic acid binding protein 2 & 2.12 & 8.63 \\
\hline ILMN_67382 & Cd63 & CD63 antigen $(\mathrm{Cd} 63)$ & 2.10 & 3.03 \\
\hline ILMN_55706 & C1s & Complement component 1, s subcomponent & 2.03 & 2.03 \\
\hline ILMN_67686 & Ms4a6b & Membrane-spanning 4-domains, subfamily $A$, member 6B & 2.03 & 2.22 \\
\hline ILMN_61063 & Rhoc & (P) Ras homolog gene family, member C & 2.02 & 2.71 \\
\hline ILMN_54242 & Vim & Vimentin & 1.96 & 2.69 \\
\hline ILMN_55431 & Rnaset2 & (P) Ribonuclease T2 & 1.88 & 1.79 \\
\hline ILMN_62651 & Eif4ebp1 & Eukaryotic translation initiation factor 4E binding protein 1 & 1.86 & 2.39 \\
\hline ILMN_58496 & C1qa & Complement component 1, q subcomponent, $\alpha$ polypeptide & 1.84 & 1.66 \\
\hline ILMN_53085 & Irf1 & Interferon regulatory factor 1 & 1.83 & 1.61 \\
\hline ILMN_62100 & Ftl1 & Ferritin light chain 1 & 1.74 & 2.28 \\
\hline ILMN_59774 & Ppp1r14b & Protein phosphatase 1, regulatory (inhibitor) subunit 14B & 1.74 & 3.36 \\
\hline ILMN_59161 & $\mathrm{Npc2}$ & Niemann Pick type $\mathrm{C} 2$ & 1.73 & 2.38 \\
\hline
\end{tabular}

(P), Predicted.

${ }^{a}$ Fold change over and above the effect of inosine of decreasing stroke-induced changes.

${ }^{b}$ Based on comparison between active regeneration state (induced by macrophage-derived factors) and normal control RGCs collected by fluorescence-activated cell sorting. From Fischer et al. (2004) (supplemental data, available at www.jneurosci.org as supplemental material).

Table 3. Complement cascade genes in LCM-captured CSMNs contralateral a stroke: induction by inosine treatment

\begin{tabular}{lll}
\hline Target & Symbol & Definition \\
\hline ILMN_58846 & Adn & (P) Adipsin \\
ILMN_55502 & C1qg & Complement component 1, q subcomponent, $\gamma$ polypeptide \\
ILMN_63608 & C2 & Complement component 2 \\
ILMN_69719 & Bf & B-factor, properdin \\
ILMN_60046 & Serping1 & Serine (or cysteine) peptidase inhibitor, clade G, member 1 \\
ILMN_51249 & C3 & (P) Complement component 3 \\
ILMN_59751 & C4-2 & (P) Complement component 4, gene 2 \\
ILMN_55706 & C1s & Complement component 1, s subcomponent \\
ILMN_58496 & C1qa & Complement component 1, q subcomponent, $\alpha$ polypeptide \\
ILMN_61448 & C1qb & Complement component 1, q subcomponent, $\beta$ polypeptide
\end{tabular}

(P), Predicted.

negative form of Mst3b abrogates the axon-promoting effects of inosine and trophic factors (Irwin et al., 2006; Lorber et al., 2007).

Other known effects of inosine may also contribute to improving outcome after stroke. Inosine has been reported to limit the size of a cortical infarct when administered before stroke, although not afterward (Shen et al., 2005), and to be neuroprotective after spinal cord injury (Liu et al., 2006). Inosine did not show obvious neuroprotective effects in our study, as judged by the absence of changes in lesion size or in caspase-3 activation. Inosine has also been reported to suppress the response of cortical neurons to glutamate (Shen et al., 2005), enhance inhibition by binding to benzodiazepine receptors (Marangos et al., 1981), limit the production of inflammatory cytokines (Haskó et al., 2000, 2004) and, at high concentrations, block hypoxia-induced astrocyte death (Haun et al., 1996; Jurkowitz et al., 1998). In addition, uric acid, a primary metabolite of inosine, prevents peroxynitrite-induced protein damage, protects the blood-brain barrier, and has potent anti-inflammatory effects (Scott et al., 2002, 2005). The extent to which these effects contributed to improving functional outcome in our study is unknown.

\section{Gene expression}

Transcriptional profiling of CSN-enriched preparations revealed that inosine affects gene expression in cells contralateral to a stroke in two ways. Inosine attenuated the many changes in gene expression induced by the stroke, while also upregulating the expression of genes related to axon growth and synaptic remodeling. The basis for the stroke-induced changes in untreated an- 


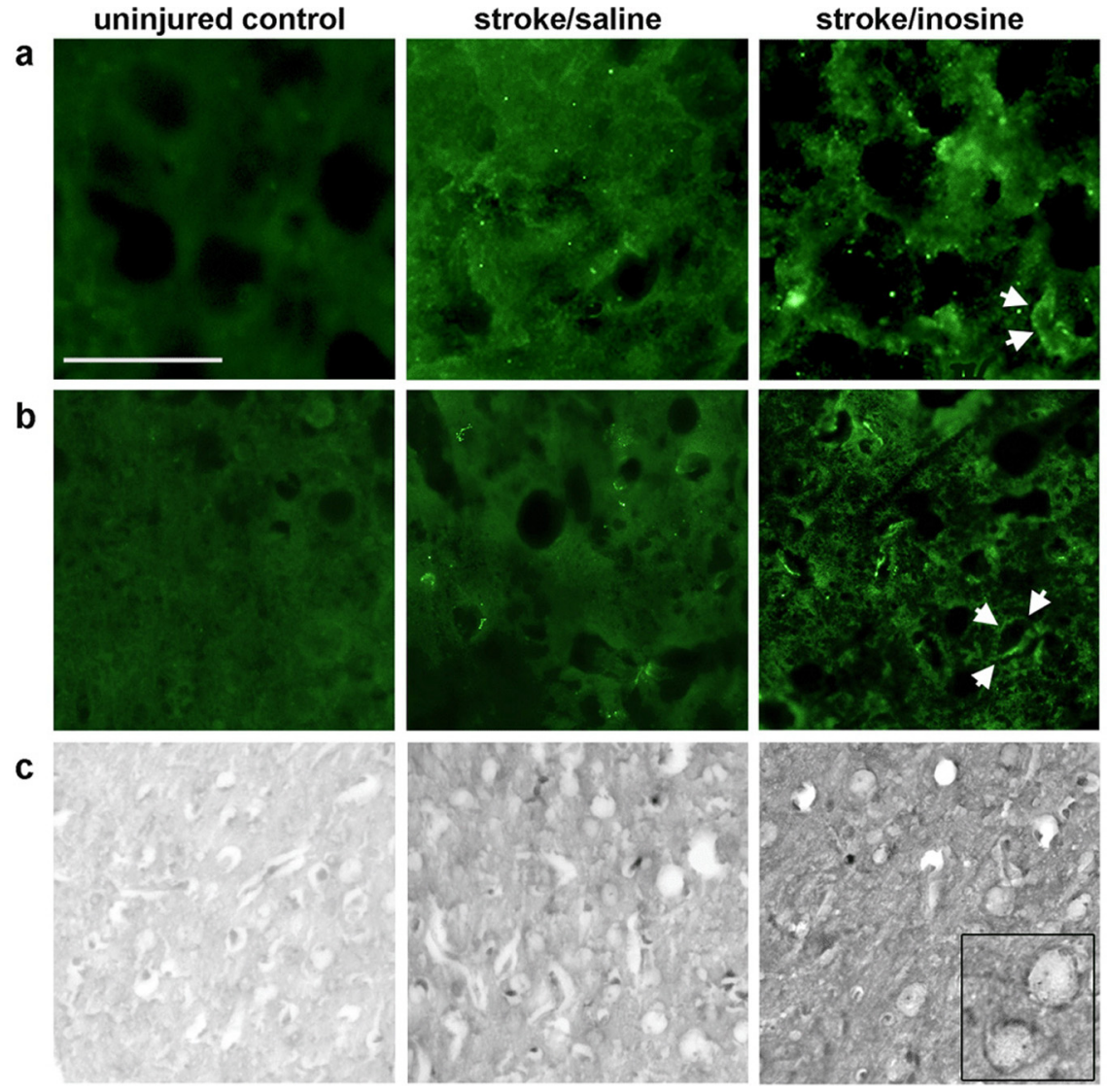

Figure 6. Inosine-induced changes in protein expression. Immunohistochemistry was used to investigate whether inosineinduced changes found at the mRNA level translate into changes at the protein level. Analyses were performed in layer 5 of the uninjured forelimb motor cortex $7 \mathrm{~d}$ after a stroke was induced in the corresponding region of the contralateral hemisphere. $\boldsymbol{a}-\boldsymbol{c}$ Inosine induced changes in levels of complement proteins $\mathrm{C}(\mathrm{q}(\boldsymbol{a})$ and $\mathrm{C}(\boldsymbol{b})$ and metallothionine $(\boldsymbol{c})$. Arrows in $\boldsymbol{a}$ and $\boldsymbol{b}$ point to outline of labeled cells; inset in c shows labeled cells at higher magnification.

imals is unknown, but could be caused by the loss of synaptic inputs from the injured side, hyperexcitability, inflammation, or hypoxia. Gene ontogeny analysis indicates that some of the stroke-induced genes are related to mitochondrial dysfunction and proteosomal processing. The ability of inosine to attenuate these changes was unanticipated, but could be related to its neuroprotective or anti-inflammatory properties. In addition to this effect, inosine increased the expression of many of the same genes that are upregulated in retinal ganglion cells undergoing axon regeneration in vivo (Fischer et al., 2004). However, inosine did not increase the expression of such well established growthassociated proteins as GAP43 and SPRR1A, perhaps reflecting differences in the molecular programs associated with collateral sprouting from undamaged neurons versus lengthy regeneration of injured axons.

In light of recent studies linking complement proteins to synaptic remodeling (Stevens et al., 2007), the upregulation of these proteins by inosine may be contributing to structural reorganization in our study. Although complement proteins have been detected in mature cortical pyramidal cells (Shen et al., 1997), their upregulation in our studies could have occurred in other cells included inadvertently in our LCM samples, e.g., microglia.

\section{Anatomical reorganization}

In adult rats, almost all CST axons decussate in the pyramids and project to the contralateral side of the spinal cord. Even in the absence of treatment, unilateral damage to the forelimb motor area significantly increased the number of CST fibers that projected from the undamaged hemisphere into the denervated side of the spinal cord. This finding is consistent with previous reports of axonal reorganization in other brain regions after stroke (Carmichael et al., 2001, 2005; Dancause et al., 2005) and other types of CNS injury (Raisman, 1969; Lynch et al., 1976; Darian-Smith and Gilbert, 1994; Buonomano and Merzenich, 1998; Z'Graggen et al., 2000; Weidner et al., 2001; Bareyre et al., 2004). Thus, the effect of inosine in enhancing CST reorganization appears to represent an augmentation of the brain's normally modest capacity to rewire itself after injury. Our results show that inosine particularly promotes the extension of long axon branches into the denervated gray matter of the spinal cord and the formation of bouton-like structures that are likely to correspond to synapses (Lagerbäck et al., 1981; Havton and Kellerth, 1987). The trajectory by which axons from the undamaged hemisphere reach the undamaged, ipsilateral side of the spinal cord is not entirely clear. Inosine did not promote CST reorganization in the absence of brain injury. These observations suggest that target denervation and/or novel patterns of brain activity seen after stroke (Carmichael, 2006) are prerequisites for neurons exposed to inosine to form new connections. Another point worth noting is that, although recent studies indicate that smaller lesions are less likely to induce dramatic anatomical reorganization than larger ones (Nudo, 2006), inosine was found here to increase axon growth even after relatively small lesions limited to the forelimb motor area and surrounding cortex. Although the lesions of the present study included most of the classical forelimb area, a more rostral area that may be important for control of the digits (Neafsey and Sievert, 1982) was not included. Hence, the recovery seen here may involve contributions from both the forelimb area of the uninjured hemisphere and spared regions of the damaged hemisphere, including perhaps the rostral forelimb area.

\section{Behavioral outcome}

Inosine enabled rats to retrieve food pellets with the impaired paw to $\sim 80 \%$ of the level seen before surgery. This task involves precise, coordinated movements of the limbs, forepaw and digits, and requires the integrity of the contralateral sensorimotor cortex for its acquisition and execution (Whishaw et al., 1993). In the absence of inosine, rats' ability to use the affected forepaw reached a plateau of $35-40 \%$ of the preoperative level of performance 3-4 weeks after stroke. Although both the spontaneous improvements and the effect of inosine in enhancing performance correlated with changes in CST organization, it is possible that the functional improvements seen here involved additional anatomical changes in both hemispheres and perhaps even other effects of inosine or uric acid, e.g., limiting neural excitability, inflammation, blood-brain barrier permeability, and protein damage. Whether inosine contributes to the changes in dendritic 
growth and neurogenesis that occur after stroke (Jones, 1999; Bury and Jones, 2002; Carmichael, 2006) remains unknown.

CST reorganization was not yet evident 2 weeks after inosine treatment. This could reflect a delay in the initiation of axon reorganization or a technical difficulty in detecting thin, nascent axons that might have been present. If CST axons arising from the ipsilateral hemisphere were not yet present at 2 weeks, the behavioral improvements seen at that time point may have been attributable to the sprouting of CST fibers arising from undamaged layer 5 pyramidal cells in the same hemisphere as the stroke, as suggested above, or to the reorganization of other pathways not studied here, or to some of the other effects of inosine suggested above.

In our previous study, rats sustained large vascular strokes in the territory of the middle cerebral artery and part of the anterior cerebral artery. This caused extensive damage to the basal ganglia and lateral cortex, and impaired extrapyramidal motor pathways and sensorimotor integration, while sparing much of the primary forelimb motor area (Chen et al., 2002). Although inosine enabled rats in that study to swipe for food pellets with the affected paw, almost none of the animals could grasp the pellets and consume them. Thus, that study left open the question of whether inosine could promote the formation of circuitry that could restore complex behaviors mediated by a specific brain area, i.e., the forepaw motor area. The differences in outcome between the previous study and this one are likely to be related primarily to differences in the extent and locus of damage. Another potentially relevant difference is that animals in the present study received a bolus injection of inosine immediately after stroke, supplementing the slow build-up in the CSF that would be expected from minipump delivery.

\section{Summary and conclusions}

Our results show that inosine alters gene expression in neurons contralateral to a photothrombotic stroke, enhances these cells' ability to form new connections on the side of the spinal cord that lost its normal innervation, and helps restore skilled behavior formerly mediated by the damaged area. In view of the established safety of inosine in other clinical settings, it may be a good candidate for use after stroke and other types of neurological damage. It will therefore be important to determine whether the results of this study can be extrapolated to other injury models in animals and to strokes in humans.

\section{References}

Allred RP, Jones TA (2004) Unilateral ischemic sensorimotor cortical damage in female rats: forelimb behavioral effects and dendritic structural plasticity in the contralateral homotopic cortex. Exp Neurol 190:433-445.

Bareyre FM, Kerschensteiner M, Raineteau O, Mettenleiter TC, Weinmann O, Schwab ME (2004) The injured spinal cord spontaneously forms a new intraspinal circuit in adult rats. Nat Neurosci 7:269-277.

Benowitz LI, Jing Y, Tabibiazar R, Jo SA, Petrausch B, Stuermer CA, Rosenberg PA, Irwin N (1998) Axon outgrowth is regulated by an intracellular purine-sensitive mechanism in retinal ganglion cells. J Biol Chem 273:29626-29634.

Buonomano DV, Merzenich MM (1998) Cortical plasticity: from synapses to maps. Annu Rev Neurosci 21:149-186.

Bury SD, Jones TA (2002) Unilateral sensorimotor cortex lesions in adult rats facilitate motor skill learning with the "unaffected" forelimb and training-induced dendritic structural plasticity in the motor cortex. J Neurosci 22:8597-8606.

Cafferty WB, Strittmatter SM (2006) The Nogo-Nogo receptor pathway limits a spectrum of adult CNS axonal growth. J Neurosci 26:12242-12250.
Carmichael ST (2003) Plasticity of cortical projections after stroke. Neuroscientist 9:64-75.

Carmichael ST (2006) Cellular and molecular mechanisms of neural repair after stroke: making waves. Ann Neurol 59:735-742.

Carmichael ST, Wei L, Rovainen CM, Woolsey TA (2001) New patterns of intracortical projections after focal cortical stroke. Neurobiol Dis 8:910-922.

Carmichael ST, Archibeque I, Luke L, Nolan T, Momiy J, Li S (2005) Growth-associated gene expression after stroke: evidence for a growthpromoting region in peri-infarct cortex. Exp Neurol 193:291-311.

Chen P, Goldberg DE, Kolb B, Lanser M, Benowitz LI (2002) Inosine induces axonal rewiring and improves behavioral outcome after stroke. Proc Natl Acad Sci U S A 99:9031-9036.

Chen S, Aston-Jones G (1995) Evidence that cholera toxin B subunit (CTb) can be avidly taken up and transported by fibers of passage. Brain Res 674:107-111.

Dancause N, Barbay S, Frost SB, Plautz EJ, Chen D, Zoubina EV, Stowe AM, Nudo RJ (2005) Extensive cortical rewiring after brain injury. J Neurosci 25:10167-10179.

Darian-Smith C, Gilbert CD (1994) Axonal sprouting accompanies functional reorganization in adult cat striate cortex. Nature 368:737-740.

Dobkin B (2003) The clinical science of neurologic rehabilitation, Ed 2. Oxford: Oxford UP.

Emerick AJ, Neafsey EJ, Schwab ME, Kartje GL (2003) Functional reorganization of the motor cortex in adult rats after cortical lesion and treatment with monoclonal antibody IN-1. J Neurosci 23:4826-4830.

Fischer D, Petkova V, Thanos S, Benowitz LI (2004) Switching mature retinal ganglion cells to a robust growth state in vivo: gene expression and synergy with RhoA inactivation. J Neurosci 24:8726-8740.

Gentleman R, Carey V, Bates B, Bolstad B, Dettling M, Dudoit S, Ellis B, Gautier L, Ge Y, Gentry J, Hornik K, Hothorn T, Huber W, Iacus S, Irizarry R, Leisch F, Li C, Maechler M, Rossini A, Sawitzki G, et al. (2004) Bioconductor: open software development for computational biology and bioinformatics. Genome Biol 5:R80.

Haskó G, Kuhel DG, Németh ZH, Mabley JG, Stachlewitz RF, Virág L, Lohinai Z, Southan GJ, Salzman AL, Szabó C (2000) Inosine inhibits inflammatory cytokine production by a posttranscriptional mechanism and protects against endotoxin-induced shock. J Immunol 164:1013-1019.

Haskó G, Sitkovsky MV, Szabó C (2004) Immunomodulatory and neuroprotective effects of inosine. Trends Pharmacol Sci 25:152-157.

Haun SE, Segeleon JE, Trapp VL, Clotz MA, Horrocks LA (1996) Inosine mediates the protective effect of adenosine in rat astrocyte cultures subjected to combined glucose-oxygen deprivation. J Neurochem 67:2051-2059.

Havton L, Kellerth JO (1987) Regeneration by supernumerary axons with synaptic terminals in spinal motoneurons of cats. Nature 325:711-714.

Hsu JE, Jones TA (2006) Contralesional neural plasticity and functional changes in the less-affected forelimb after large and small cortical infarcts in rats. Exp Neurol 201:479-494.

Irwin N, Li YM, O’Toole JE, Benowitz LI (2006) Mst3b, a purine-sensitive Ste20-like protein kinase, regulates axon outgrowth. Proc Natl Acad Sci U S A 103:18320-18325.

Jones TA (1999) Multiple synapse formation in the motor cortex opposite unilateral sensorimotor cortex lesions in adult rats. J Comp Neurol 414:57-66.

Jurkowitz MS, Litsky ML, Browning MJ, Hohl CM (1998) Adenosine, inosine, and guanosine protect glial cells during glucose deprivation and mitochondrial inhibition: correlation between protection and ATP preservation. J Neurochem 71:535-548.

Kawamata T, Dietrich WD, Schallert T, Gotts JE, Cocke RR, Benowitz LI, Finklestein SP (1997) Intracisternal basic fibroblast growth factor enhances functional recovery and up-regulates the expression of a molecular marker of neuronal sprouting following focal cerebral infarction. Proc Natl Acad Sci U S A 94:8179-8184.

Lagerbäck PA, Ronnevi LO, Cullheim S, Kellerth JO (1981) An ultrastructural study of the synaptic contacts of alpha-motoneurone axon collaterals. I. Contacts in lamina IX and with identified alpha-motoneurone dendrites in lamina VII. Brain Res 207:247-266.

Lee JK, Kim JE, Sivula M, Strittmatter SM (2004) Nogo receptor antagonism promotes stroke recovery by enhancing axonal plasticity. J Neurosci 24:6209-6217.

Liu F, You SW, Yao LP, Liu HL, Jiao XY, Shi M, Zhao QB, Ju G (2006) 
Secondary degeneration reduced by inosine after spinal cord injury in rats. Spinal Cord 44:421-426.

Lorber B, Howe ML, Benowitz LI, Irwin N (2008) Mst3b, an Ste20-like kinase, regulates axon regeneration in the mature CNS and PNS. Soc Neurosci Abstr 33:1376.

Luke LM, Allred RP, Jones TA (2004) Unilateral ischemic sensorimotor cortical damage induces contralesional synaptogenesis and enhances skilled reaching with the ipsilateral forelimb in adult male rats. Synapse 54:187-199.

Lynch G, Gall C, Rose G, Cotman C (1976) Changes in the distribution of the dentate gyrus associational system following unilateral or bilateral entorhinal lesions in the adult rat. Brain Res 110:57-71.

Marangos PJ, Trams E, Clark-Rosenberg RL, Paul SM, Skolnick P (1981) Anticonvulsant doses of inosine result in brain levels sufficient to inhibit [3H] diazepam binding. Psychopharmacology 75:175-178.

Markgraf CG, Kraydieh S, Prado R, Watson BD, Dietrich WD, Ginsberg MD (1993) Comparative histopathologic consequences of photothrombotic occlusion of the distal middle cerebral artery in Sprague-Dawley and Wistar rats. Stroke 24:286-292; discussion 292-293.

Neafsey EJ, Sievert C (1982) A second forelimb motor area exists in rat frontal cortex. Brain Res 232:151-156.

Nudo RJ (2006) Mechanisms for recovery of motor function following cortical damage. Curr Opin Neurobiol 16:638-644.

Nudo RJ (2007) Postinfarct cortical plasticity and behavioral recovery. Stroke 38:840-845.

Papadopoulos CM, Tsai SY, Alsbiei T, O’Brien TE, Schwab ME, Kartje GL (2002) Functional recovery and neuroanatomical plasticity following middle cerebral artery occlusion and IN-1 antibody treatment in the adult rat. Ann Neurol 51:433-441.

Papadopoulos CM, Tsai SY, Cheatwood JL, Bollnow MR, Kolb BE, Schwab ME, Kartje GL (2006) Dendritic plasticity in the adult rat following middle cerebral artery occlusion and Nogo-a neutralization. Cereb Cortex 16:529-536.

Paxinos G, Watson C (1998) The rat brain in stereotaxic coordinates, Ed 4. New York: Academic.

Raisman G (1969) Neuronal plasticity in the septal nuclei of the adult rat. Brain Res 14:25-48.
Scott GS, Spitsin SV, Kean RB, Mikheeva T, Koprowski H, Hooper DC (2002) Therapeutic intervention in experimental allergic encephalomyelitis by administration of uric acid precursors. Proc Natl Acad Sci U S A 99:16303-16308.

Scott GS, Cuzzocrea S, Genovese T, Koprowski H, Hooper DC (2005) Uric acid protects against secondary damage after spinal cord injury. Proc Natl Acad Sci U S A 102:3483-3488.

Shen H, Chen GJ, Harvey BK, Bickford PC, Wang Y (2005) Inosine reduces ischemic brain injury in rats. Stroke 36:654-659.

Shen Y, Li R, McGeer EG, McGeer PL (1997) Neuronal expression of mRNAs for complement proteins of the classical pathway in Alzheimer brain. Brain Res 769:391-395.

Smith JM, Lunga P, Story D, Harris N, Le Belle J, James MF, Pickard JD, Fawcett JW (2007) Inosine promotes recovery of skilled motor function in a model of focal brain injury. Brain 130:915-925.

Smyth GK (2005) Limma: linear models for microarray data. In: Bioinformatics and computational biology solutions using $\mathrm{R}$ and bioconductor (Gentleman R, Carey V, Huber W, Irizarry R, Dudoit S, eds), pp 397-420. New York: Springer.

Stevens B, Allen NJ, Vazquez LE, Howell GR, Christopherson KS, Nouri N, Micheva KD, Mehalow AK, Huberman AD, Stafford B, Sher A, Litke AM, Lambris JD, Smith SJ, John SW, Barres BA (2007) The classical complement cascade mediates CNS synapse elimination. Cell 131:1164-1178.

Weidner N, Ner A, Salimi N, Tuszynski MH (2001) Spontaneous corticospinal axonal plasticity and functional recovery after adult central nervous system injury. Proc Natl Acad Sci U S A 98:3513-3518.

Whishaw IQ, Pellis SM, Gorny B, Kolb B, Tetzlaff W (1993) Proximal and distal impairments in rat forelimb use in reaching follow unilateral pyramidal tract lesions. Behav Brain Res 56:59-76.

Z'Graggen WJ, Fouad K, Raineteau O, Metz GA, Schwab ME, Kartje GL (2000) Compensatory sprouting and impulse rerouting after unilateral pyramidal tract lesion in neonatal rats. J Neurosci 20:6561-6569.

Zurn AD, Do KQ (1988) Purine metabolite inosine is an adrenergic neurotrophic substance for cultured chicken sympathetic neurons. Proc Natl Acad Sci U S A 85:8301-8305. 